\title{
Pearls \& Oy-sters: Persistent elevation of serum carcinoembryonic antigen in secretory meningioma
}

Mauricio F. Villamar, MD, Farhan A. Mirza, MBBS, Jonathan H. Smith, MD, and Vanessa D. Smith, MD

Neurology ${ }^{\circledR}$ 2018;90:e538-e541. doi:10.1212/WNL.0000000000004934
Correspondence

Dr. Smith

vmdima2@gmail.com

\section{Pearls}

- Elevation in serum carcinoembryonic antigen (CEA) is uncommon in the context of primary CNS neoplasms but can be seen with metastatic brain tumors. CEA elevation can be the presenting feature of a secretory meningioma (SM), a rare subtype of WHO grade I meningioma.

- Despite its rarity, SM is a highly relevant entity as it is frequently associated with severe peritumoral brain edema, which can lead to perioperative morbidity and mortality.

\section{Oy-sters}

- SM can present diagnostic and management pitfalls for neurologists, neurosurgeons, and pathologists.

- In patients with oncologic history presenting with brain tumors, the possibility of nonmetastatic etiologies should always be considered.

\section{Case report}

A 73-year-old woman with stage 3B chronic kidney disease (CKD) and remote history of tobacco use was diagnosed with stage 1 (nonmetastatic) colon cancer on a screening colonoscopy. The tumor was discovered in the proximal ascending colon. She underwent laparoscopic right hemicolectomy and did not require any adjunctive chemotherapy or radiation. At the time of her initial diagnosis, serum CEA level was marginally elevated at $3.1 \mathrm{ng} / \mathrm{mL}$ (reference range $0-3.0 \mathrm{ng} / \mathrm{mL}$ ). Five years later, despite being asymptomatic with stable renal function, her serum CEA level was elevated at $8.2 \mathrm{ng} / \mathrm{mL}$. This prompted a CT with contrast of the abdomen and pelvis and repeat colonoscopy, which did not reveal evidence of cancer recurrence. At age 79, she presented with a marked decline in her overall health over the preceding 3-6 months, with weight loss, frequent headaches, worsening cognition, and difficulty performing activities of daily living.

On admission, the patient's general physical and neurologic examination results were unremarkable. Laboratory workup revealed acute on CKD, hyperkalemia, and uremia secondary to dehydration. A CT head without contrast demonstrated a large right frontal mass with extensive peritumoral edema, mass effect, and 12 -mm leftward midline shift (figure). Brain MRI, performed without gadolinium due to her renal impairment, confirmed a right frontal lobe mass with extensive perilesional edema and associated mass effect (figure), with 10-mm leftward midline shift and subfalcine herniation. CT without contrast of the chest, abdomen, and pelvis, obtained due to her history of colon cancer, showed no evidence of malignancy or lymphadenopathies. Serum CEA level was elevated at $13.6 \mathrm{ng} / \mathrm{mL}$.

A right frontal craniotomy was performed and gross total resection of the tumor was achieved. Microscopic examination revealed a neoplasm with lobular architecture composed of round, uniform nuclei with occasional intranuclear pseudoinclusions, growing in a syncytial pattern. Focal areas demonstrated whorling architecture. There were, in addition, numerous gland-like

From the Departments of Neurology (M.F.V., J.H.S.), Neurosurgery (F.A.M.), and Pathology and Laboratory Medicine (V.D.S.), University of Kentucky, Lexington.

Go to Neurology.org/N for full disclosures. Funding information and disclosures deemed relevant by the authors, if any, are provided at the end of the article. 


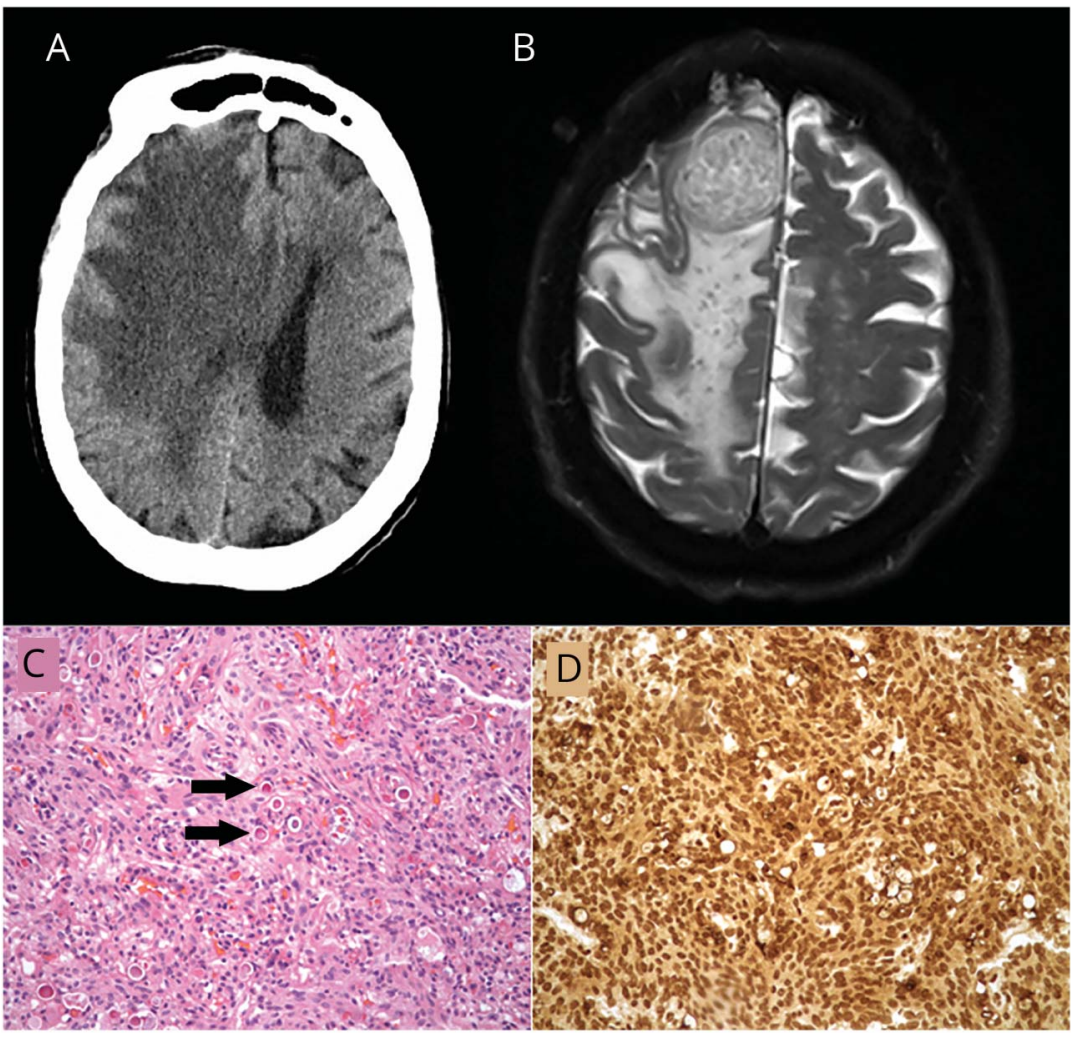

(A) CT head without contrast demonstrates a large right frontal mass with extensive peritumoral edema, mass effect, and 12-mm leftward midline shift. (B) Brain MRI without gadolinium (T2-weighted) confirms a right frontal lobe mass with extensive perilesional edema and associated mass effect. (C) Low-power (×10) image of hematoxylin \& eosin-stained tissue demonstrates a neoplasm composed of round, uniform nuclei with occasional intranuclear pseudoinclusions. The architecture is predominantly lobular with focal areas of whorling. Numerous gland-like lumina filled with eosinophilic and granular secretions are seen (arrows). (D) Low-power $(\times 10)$ image of immunohistochemical staining with carcinoembryonic antigen demonstrates strong and diffuse immunoreactivity within both the neoplastic cells and luminal secretions.

lumina filled with eosinophilic and granular secretions present throughout the tissue, closely mimicking the appearance of psammoma bodies (figure). Immunohistochemical staining demonstrated positivity for CEA (figure) as well as for epithelial membrane antigen, a nonspecific marker that can occasionally show positivity in meningiomas. In conjunction with the morphologic findings, the diagnosis was consistent with a secretory meningioma. This was supported by clinical findings of extensive peritumoral edema on MRI and elevated serum CEA levels.

Postoperatively, the patient was noted to have left hemiparesis that resolved within 24 hours.

Postoperative MRI without gadolinium demonstrated gross total resection of the tumor. There were small areas of restricted diffusion within the right posterior frontal and parietal lobes, adjacent to the operative bed. The patient was discharged to a subacute rehabilitation facility. She was lost to follow-up.

\section{Discussion}

CEA is a cell adhesion glycoprotein produced during fetal development. Although production typically ceases at birth, elevated serum CEA level can occur in multiple neoplasms, most notably in gastrointestinal and gynecologic malignancies. However, elevated serum level can also be seen in states such as nicotine dependence, $\mathrm{CKD}$, liver cirrhosis, pneumonia, diabetes mellitus, and many others. Its clinical use in cancer screening is therefore controversial. ${ }^{1}$

Serum CEA can also be elevated in CNS malignancies. In a study by Suzuki and Tanaka, ${ }^{2}$ mild elevations in serum CEA were present in $4 \%$ of primary brain tumors, never exceeding $4.0 \mathrm{ng} / \mathrm{mL}$. Conversely, $37 \%$ of patients with metastatic brain tumors had elevated serum CEA levels regardless of their primary neoplasm. The majority had concentrations greater than $5.0 \mathrm{ng} / \mathrm{mL}$. Pronounced elevation in serum CEA in the context of a primary brain tumor, whether parenchymal or meningeal, is uncommon.

First reported by Alguacil-García et al. ${ }^{3}$ in 1986, SM is a very rare subtype of WHO grade I meningioma. SM has been estimated to account for $1 \%-3 \%$ of all meningiomas. It most often affects individuals in the fifth to the seventh decades of age and has a high female:male ratio, consistent with most meningioma subtypes. ${ }^{4-6}$ As with other CNS neoplasms, clinical manifestations of SM depend on tumor location and extension. Nevertheless, seizures appear to be more common in SM than in nonsecretory meningiomas. ${ }^{7}$ Short- and long-term prognosis of SM depend on tumor location and extent of resection. ${ }^{6}$

Pathologically, one of the hallmarks of SM is the presence of pseudopsammoma bodies, which are glandular lumina with 
eosinophilic and periodic acid-Schiff (PAS)-positive secretory globules. Both these globules and the cells surrounding them show strong CEA positivity and often immunoreactivity to cytokeratin, an epithelial marker uncommonly positive in meningiomas. ${ }^{3} \mathrm{SM}$ can be a diagnostic pitfall for pathologists as its appearance can mimic epithelial neoplasms, such as metastatic carcinoma, particularly if given a small biopsy sample or if the pathologist is inexperienced in neuropathology. Consistent with their female predominance, $33 \%-100 \%$ of SM also stain positive for progesterone and estrogen receptors. ${ }^{4}$ Other areas of the tumor show classic meningioma histology.

Despite its benign pathology, one exceptional characteristic that differentiates SM from other grade 1 meningioma subtypes is its association with severe peritumoral edema. In most cases of SM, radiologically estimated peritumoral brain edema exceeds the size of the actual tumor. ${ }^{4,6}$ Edema can involve the majority of the affected hemisphere in as many as $40 \%$ of cases. ${ }^{4}$ Perioperative surgical and nonsurgical complications, largely related to cerebral edema, could be twice as common in SM as compared to nonsecretory meningiomas. ${ }^{7}$

The extent of cerebral edema related to SM has been associated with the number of PAS (+), CEA $(+)$, and cytokeratin $(+)$ cells within the tumor. ${ }^{4}$ In SM, there are also high concentrations of mast cells in and around pseudopsammoma bodies. ${ }^{5}$ Secretion of proinflammatory and proangiogenic mediators such as histamine, serotonin, and vascular endothelial growth factor (VEGF) by mast cells can play a major role in the development of peritumoral edema in $\mathrm{SM}^{8,9}$

As noted above, pseudopsammoma bodies often stain positive for CEA when immunohistochemistry is performed in SM tissue samples. However, elevated serum CEA levels are only present in 50\%-60\% of patients with SM. ${ }^{6}$ Furthermore, the clinical scenario that was seen in our patient, a diagnosis of SM in the setting of a history of colon cancer, is exceedingly rare and represents one of the highlights of the present article. To our knowledge, there is only one similar case report. A 60 -year-old woman was diagnosed with nonmetastatic breast cancer. Serum CEA was $23 \mathrm{ng} / \mathrm{mL}$ at the time of diagnosis. Despite subtotal mastectomy with negative axillary node dissection and multiple negative reevaluations for metastatic disease, serum CEA levels remained elevated (13-18 $\mathrm{ng} / \mathrm{mL})$ for 2 years until she was diagnosed with a sphenoid wing SM. CEA levels normalized following SM resection. ${ }^{10}$

SM is a diagnostic challenge as it can simulate metastatic neoplasms clinically, radiologically, and pathologically. In our patient, her history of colon cancer, the persistent elevation in serum CEA, and the radiographic appearance of her brain lesion were all highly suggestive of a dural-based metastatic tumor. Importantly, CEA levels can be increased in individuals with CKD. ${ }^{1}$ However, our patient's glomerular filtration rate remained stable around $40 \mathrm{~mL} / \mathrm{min}$ for approximately 5 years. Simultaneously, her serum CEA levels progressively increased from 3.1 to $8.2 \mathrm{ng} / \mathrm{mL}$, suggesting that such elevation was secondary to a different pathophysiologic process.

SM can often be diagnosed at the time of frozen sections. Therefore, whenever neuroimaging reveals a meningeal-based tumor associated with perilesional edema that exceeds the size of the actual tumor, intraoperative frozen sections may be considered. $^{4}$

Pretreatment with IV steroids can decrease perilesional edema and intracranial pressure (ICP) prior to surgery. Intraoperatively, common measures for reducing ICP include head elevation in relation to the heart, prevention of jugular vein compression after patient positioning to avoid outflow obstruction, use of mannitol \pm furosemide for osmotic diuresis, gentle manipulation of the lesion and brain tissue with microneurosurgical dissection techniques, and preservation of venous structures to prevent postoperative venous congestion. Postoperatively, head of bed elevation, continuation of IV steroids, and early immobilization, if possible, should be considered. VEGF-directed therapies are experimental. ${ }^{9} \quad$ H2-receptor antagonists (i.e., famotidine) are frequently used to prevent gastrointestinal side effects of steroids. However, their antihistaminergic properties could theoretically assist in the management of peritumoral edema related to SM. Although our patient did not experience major perioperative complications, the severe peritumoral edema that accompanies SM can be challenging to manage.

In patients who are found to have a dural-based lesion with extensive vasogenic edema, SM should be included in the differential as prompt and accurate diagnosis of this entity can better direct both medical and surgical management. There are no clinically useful serum tumor markers whose presence can help identify primary brain tumors, in part because of limited passage of molecules across the blood-brain barrier. Some exceptions include elevated $\alpha$-fetoprotein and human chorionic gonadotropin levels in certain midline/pineal germ cell tumors. Although pituitary adenomas can show elevations in endocrine hormones, serum levels do not necessarily correlate with staining of the tumor itself. Efforts to measure biomarkers of certain primary brain tumor markers in CSF are underway. ${ }^{11}$

\section{Author contributions}

Mauricio F. Villamar: case concept and design, acquisition of data, interpretation of data, manuscript writing. Farhan A. Mirza: case concept and design, acquisition of data, interpretation of data, manuscript writing. Jonathan $\mathrm{H}$. Smith: case concept and design, interpretation of data, critical revision of manuscript for intellectual content. Vanessa D. Smith: case concept and design, interpretation of data, critical revision of manuscript for intellectual content. 


\section{Study funding}

No targeted funding reported.

\section{Disclosure}

The authors report no disclosures relevant to the manuscript. Go to Neurology.org/N for full disclosures.

\section{References}

1. Nicholson BD, Shinkins B, Pathiraja I, et al. Blood CEA levels for detecting recurrent colorectal cancer. Cochrane Database Syst Rev 2015:CD011134.

2. Suzuki Y, Tanaka R. Carcinoembryonic antigen in patients with intracranial tumors. J Neurosurg 1980;53:355-360.

3. Alguacil-García A, Pettigrew NM, Sima AA. Secretory meningioma: a distinct subtype of meningioma. Am J Surg Pathol 1986;10:102-111.

4. Regelsberger J, Hagel C, Emami P, Ries T, Heese O, Westphal M. Secretory meningiomas: a benign subgroup causing life-threatening complications. Neuro Oncol 2009;11:819-824.
5. Tirakotai W, Mennel HD, Celik I, Hellwig D, Bertalanffy H, Riegel T. Secretory meningioma: immunohistochemical findings and evaluation of mast cell infiltration. Neurosurg Rev 2006;29:41-48.

6. Wang DJ, Xie Q, Gong Y, et al. Secretory meningiomas: clinical, radiological and pathological findings in 70 consecutive cases at one institution. Int J Clin Exp Pathol 2013;6:358-374.

7. Mohme M, Emami P, Regelsberger J, et al. Secretory meningiomas: increased prevalence of seizures secondary to edema formation in a rare histologic subtype. World Neurosurg 2016;92:418-425.

8. Nagy JA, Benjamin L, Zeng H, Dvorak AM, Dvorak HF. Vascular permeability, vascular hyperpermeability and angiogenesis. Angiogenesis 2008;11:109-119.

9. Hou J, Kshettry VR, Selman WR, Bambakidis NC. Peritumoral brain edema in intracranial meningiomas: the emergence of vascular endothelial growth factor-directed therapy. Neurosurg Focus 2013;35:E2.

10. Louis DN, Hamilton AJ, Sobel RA, Ojemann RG. Pseudopsammomatous meningioma with elevated serum carcinoembryonic antigen: a true secretory meningioma. Case report. J Neurosurg 1991;74:129-132.

11. Staedtke V, Dzaye ODA, Holdhoff M. Actionable molecular biomarkers in primary brain tumors. Trends Cancer 2016;2:338-349. 


\section{Neurology}

\section{Pearls \& Oy-sters: Persistent elevation of serum carcinoembryonic antigen in secretory meningioma}

Mauricio F. Villamar, Farhan A. Mirza, Jonathan H. Smith, et al. Neurology 2018;90;e538-e541

DOI 10.1212/WNL.0000000000004934

\section{This information is current as of February 5, 2018}

\section{Updated Information \&} Services

References

Subspecialty Collections

\section{Permissions \& Licensing}

Reprints including high resolution figures, can be found at: http://n.neurology.org/content/90/6/e538.full

This article cites 10 articles, 0 of which you can access for free at: http://n.neurology.org/content/90/6/e538.full\#ref-list-1

This article, along with others on similar topics, appears in the following collection(s):

All Oncology

http://n.neurology.org/cgi/collection/all_oncology

Critical care

http://n.neurology.org/cgi/collection/critical_care

Metastatic tumor

http://n.neurology.org/cgi/collection/metastatic_tumor

Primary brain tumor

http://n.neurology.org/cgi/collection/primary_brain_tumor

Surgical therapy-tumor

http://n.neurology.org/cgi/collection/surgical_therapytumor

Information about reproducing this article in parts (figures,tables) or in its entirety can be found online at:

http://www.neurology.org/about/about_the_journal\#permissions

Information about ordering reprints can be found online:

http://n.neurology.org/subscribers/advertise

Neurology ${ }^{\circledR}$ is the official journal of the American Academy of Neurology. Published continuously since 1951, it is now a weekly with 48 issues per year. Copyright (C) 2018 American Academy of Neurology. All rights reserved. Print ISSN: 0028-3878. Online ISSN: 1526-632X.

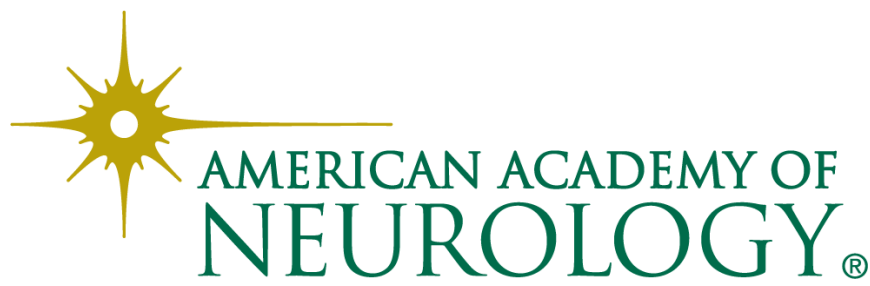

